\title{
GUALIdAde Física, MicRobiológica e PARAsitológica de ALFACES (Lactuca sativa) DE DIFERENTES SISTEMAS DE CULTIVO'
}

\author{
Ligia Regina R. de SANTANA², Rosemary D.S. CARVALHO ${ }^{3}$, Clicia Capibaribe LEITE ${ }^{3}$, \\ Leda Maria ALCÂNTARA ${ }^{3}$, Tchana Weyll S. de OLIVEIRA ${ }^{4}$, Breno da M. RODRIGUES ${ }^{3}$
}

\begin{abstract}
RESUMO
A alface (Lactuca sativa) é a hortaliça folhosa mais comercializada no Brasil. Seu baixo valor calórico a qualifica para diversas dietas, o que favorece o seu consumo sob a forma crua, possibilitando a ocorrência de enfermidades intestinais. O objetivo deste trabalho foi realizar uma avaliação física, microbiológica e parasitológica de amostras de alfaces, variedade crespa, provenientes dos sistemas de cultivo orgânico, tradicional e hidropônico, comercializadas nos principais supermercados da cidade de Salvador (BA), no período de setembro de 2003 a junho de 2004. As amostras do cultivo hidropônico apresentaram o menor peso total e da parte bio-comestível, diferindo significativamente $(\mathrm{p}<0,05)$ das amostras de cultivo orgânico e tradicional; foram classificadas como Extra, de acordo com a legislação vigente, enquanto que as de cultivo orgânico e tradicional foram inseridas como hortaliças De Primeira e De Segunda classe, respectivamente. As amostras de alfaces, independente do sistema de cultivo, apresentaram baixos padrões higiênicos, indicados pela presença de formas parasitológicas de origem animal ou humana e alta concentração de coliformes fecais, sendo que foram observadas as maiores freqüências de contaminação nas amostras do cultivo orgânico, seguida das de cultivo tradicional e hidropônico. Considerando-se os resultados obtidos, ressalta-se a importância desta hortaliça na transmissão de enfermidades intestinais, assim como a necessidade de medidas que propiciem uma melhoria na sua qualidade higiênico-sanitária.
\end{abstract}

Palavras-chave: Lactuca sativa, alface, qualidade microbiológica, parasitológica, física.

\section{SUMMARY}

PHYSICAL, MICROBIOLOGICAL AND PARASITOLOGICAL QUALITY OF LETTUCE (Lactuca sativa) FROM DIFFERENT GROWING PROCESSES. The lettuce (Lactuca sativa) has economic importance to the people in Brazil. The aim of this study was to carry out a physical, microbiological and parasitological evaluations in the lettuce samples - crisp variety, according to organic, traditional and hydroponics growing processes, commercialized at the supermarkets of the City of Salvador, Bahia, Brazil, collected from September/2003 until June/2004. The hydroponics lettuce samples showed the lowest total and edible part weight; they were inserted into Extra rate quality standard, according to the current legislation, whereas the organic and traditional lettuce samples were inserted into First and Second rate quality standard, respectively. The lettuce samples, independently of the growing process, showed low hygienic conditions, indicated by the presence of parasites of animal or human origin, and high concentration of fecal coliforms; the organic lettuce samples showed the highest frequencies of enteroparasities and fecal coliforms concentration, followed by the traditional and hydroponics lettuce samples. Considering the obtained results, the importance of this kind of food in the transmission of enteroparasities is stressed, as well as the need of actions which improve the sanitary conditions of these products.

Keywords: Lactuca sativa, lettuce, microbiological, parasitilogical, physical quality.

\section{1 - INTRODUÇÃO}

A alface (Lactuca sativa) é a hortaliça folhosa mais comercializada no Brasil, sendo considerada uma cultura hortícola de grande consumo. Devido ao seu baixo valor calórico qualifica-se para diversas dietas, o que favorece grandemente o seu consumo de uma maneira geral, constituindo-se em componente imprescindível das saladas dos brasileiros [6].

${ }^{1}$ Recebido para publicação em 19/7/2004 Aceito para publicação em 28/04/2006 (001379)

${ }^{2}$ Departamento de Ciências da Vida, curso de Nutrição da Universidade do Estado da Bahia (Uneb)

Estrada das Barreiras, $s / n^{\circ}$, Narandiba - Cabula

CEP 41195-001 - Salvador (BA)

E-mail: ligiarrs@ig.com.br

${ }^{3}$ Faculdade de Farmácia da Universidade Federal da Bahia (UFBA)

Rua Barão de Geremoabo, $s / n^{\circ}$, Campus de Ondina

CEP 40170-210 - Salvador (BA)

E-mail:clicia@ufba.br

${ }^{4}$ Bolsista PICIN - Departamento de Ciências da Vida, curso de Nutrição da Uneb

E-mail: tchw@ig.com.br
Dados da comercialização no mercado atacadista Ebal/Ceasa (Empresa Baiana de Alimentos) revelaram que o Estado da Bahia comercializou, no ano de 2003, cerca de $518.487,50 \mathrm{~kg}$ de alface, com um valor comercial de R\$ 1.237.995,86. Deste montante comercializado, apenas 137.860,50 Kg do produto são oriundos do Estado da Bahia, com destaque para as regiôes de Jaguaquara, Morro do Chapéu, Amélia Rodrigues e Conceição do Jacuípe; sendo o restante fornecido pelos Estados de São Paulo (365.482,84 $\mathrm{Kg})$ e de Sergipe (15.144,16 Kg).

O cultivo da alface vem sendo praticado na forma tradicional, hidropônica e orgânica, que apresentam características diferenciadas na produção, podendo influenciar nas propriedades desta hortaliça [18]. A agricultura orgânica surge como alternativa de produção à agricultura altamente mecanizada e rica em insumos industriais, que caracterizam o cultivo tradicional. O cultivo orgânico pode ser definido como "sistema de produção que evita ou exclui o uso de pesticidas ou agrotóxicos, fertilizantes de composição sintética, reguladores de crescimento ou 
outros agentes contaminantes. A sua viabilização é através de um conjunto de sistemas de produção, buscando a maximização dos benefícios sociais, a auto-sustentação, a redução/eliminação da dependência de insumos, energia não renovável e a preservação do meio ambiente através da otimização do uso de recursos naturais e sócio-econômicos disponíveis" [10].

O cultivo hidropônico protege a hortaliça contra fatores adversos do meio ambiente, como chuvas, geadas e ventos fortes e outros, favorecendo a produtividade do produto. As hortaliças hidropônicas devem, necessariamente, receber nutrientes previamente dissolvidos em água, pois são cultivadas fora de seu ambiente natural (terra), em tubos plásticos, por onde circula a água contendo os fertilizantes químicos [2].

Nos últimos anos, o interesse por produtos oriundos da agricultura agroecológica vem crescendo intensamente. Conseqüentemente, o consumidor tem uma possibilidade de escolha ampliada nos pontos de venda das grandes cidades, podendo optar pelo produto orgânico, hidropônico ou convencional. No entanto, a não ser pelas diferenças práticas entre os três tipos de cultivo, o consumidor não possui dados indicativos sobre a qualidade e as propriedades das hortaliças oriundas dos diferentes cultivos [18].

As hortaliças, em especial, as consumidas cruas, necessitam ser puras e saudáveis, sendo estas, exigências crescentes da sociedade. No entanto, possibilitam ocorrência de enfermidades intestinais, uma vez que helmintos, protozoários e outros patógenos podem estar presentes nessas verduras, que são freqüentemente adubadas e/ ou irrigadas com água contaminada por dejetos fecais. As doenças transmitidas por alimentos são, predominantemente, resultantes do ciclo de contaminação fecal/oral e seu controle deve receber atenção cada vez maior em nosso meio [16, 17].

No Brasil, não obstante a relevância e atualidade do problema, são poucos os trabalhos que avaliam a qualidade das hortaliças consumidas pela população. Assim, o diagnóstico laboratorial de protozoários e helmintos parasitas de humanos e demais patógenos em hortaliças é de grande importância para a saúde pública, uma vez que fornecem dados sobre as condições higiênicas envolvidas na produção, armazenamento, transporte e manuseio desses produtos e, permite o controle retrospectivo das condições em que foram cultivadas [23, 7].

Com base nestes aspectos e considerando, sobretudo, a carência de informações comparativas sobre os níveis de contaminação dos produtos oriundos do cultivo tradicional, hidropônico e orgânico, este trabalho teve como objetivo a avaliação física, microbiológica e parasitológica de amostras de alfaces, variedade crespa, provenientes destes três sistemas de cultivo, comercializadas nos principais supermercados da cidade de Salvador (BA).

\section{2 - MATERIAL E MÉTODOS}

\section{1 - Amostra}

Foram utilizadas 60 amostras de alface (Lactuca sativa), variedade crespa, de cada sistema de cultivo (tradicional, hidropônico, orgânico), considerando-se os estudos com hortaliças de diversos autores do país [ 16, 7], perfazendo um total de 180 amostras. Estabeleceu-se como unidade amostral, um pé ou cabeça de alface, independentemente do seu peso ou tamanho, adotando-se como critério que cada amostra apresentasse boa qualidade e características organolépticas visuais próprias.

\section{2 - Coleta das amostras}

Realizada nos meses de setembro de 2003 a junho de 2004, período em que a irrigação artificial é utilizada com maior intensidade devido aos menores índices pluviométricos no Estado da Bahia. A coleta ocorreu no período da manhã, visitando-se dois ou três supermercados da cidade de Salvador (BA) e retirando-se, aleatoriamente, uma amostra de alface fresca, in natura, inteira, do lote de hortaliças a venda, (de acordo com a RDC-12, Anexo I, enquadra-se no Grupo 2.a), segundo cada sistema de cultivo. As amostras em seus respectivos invólucros foram acondicionadas individualmente em sacos de polietileno de primeiro uso, sem contato manual, devidamente identificadas e levadas ao laboratório para análise.

\section{3 - Avaliação física}

Todas as amostras de alface foram avaliadas semanalmente quanto à aparência, conforme a legislação vigente [1], recebendo pontuação que variou de 1 a 3 , segundo a descrição exposta abaixo:

1) Extra - quando constituídas por hortaliças de elevada qualidade, bem desenvolvidas, compactas e firmes. Não sendo permitidos defeitos nas hortaliças desta classe;

2) De Primeira - quando forem de boa qualidade, bem desenvolvidas, compactas e firmes. As hortaliças deverão apresentar coloração uniforme, típica da variedade. Não sendo permitidos danos, que alterem sua conformação e aparência, contudo, são tolerados pequenos defeitos ou manchas. Não são permitidas rachaduras, cortes e perfurações;

3) De Segunda - quando constituídas por hortaliças que não foram classificadas nas classes anteriores. São tolerados pequenos defeitos na conformação e ligeira descoloração desde que não afetem seriamente as suas características. São também tolerados pequenos danos de origem física ou mecânica, desde que não causem defeitos graves.

Para a determinação do peso total, as amostras foram pesadas individualmente, utilizando-se balança semi-analítica; em seguida, foram separadas as folhas recolhendo-as em sacos de polietileno estéreis, desprezando-se aquelas manchadas ou deterioradas, bem como o talo, obtendo-se o peso da parte bio-comestível [13]. Para o manuseio das amostras, utilizaram-se luvas descartáveis. 


\section{4 - Avaliação microbiológica}

A análise microbiológica foi realizada na água proveniente das duas lavagens ocorridas nas amostras. Para a primeira lavagem, foram adicionados $500 \mathrm{~mL}$ de água destilada às folhas de alface contidas em sacos plásticos estéreis, as quais foram agitadas manualmente por $60 \mathrm{~s}$. Após a retirada da primeira água de lavagem, que seguiu para análises, foram adicionados $500 \mathrm{~mL}$ de solução ácida (água $+30 \%$ de vinagre comum) nas amostras, as quais foram deixadas em repouso por $10 \mathrm{~min}$ [14].

Foram coletados em frascos estéreis, $200 \mathrm{~mL}$ da água de ambas as lavagens, sendo determinado o número mais provável (NMP) de bactérias dos grupos coliformes totais $\left(35^{\circ} \mathrm{C}\right)$ e coliformes fecais $\left(45^{\circ} \mathrm{C}\right)$ e pesquisa de Salmonella spp. (em $25 \mathrm{~mL}$ de amostra), segundo metodologia descrita por VANDERZANT \& SPLITTSTOESSER [24].

\section{5 - Avaliação microscópica}

Foi realizada pesquisa de sujidades leves (ácaros, larvas, insetos, fragmentos de insetos) na água proveniente das duas lavagens ocorridas nas amostras de alface, de acordo com método recomendado pela A.O.A.C. [3].

A análise parasitológica também foi realizada após as duas lavagens. Assim, para cada água de lavagem foi adotado o seguinte procedimento: foi distribuída em tubos e centrifugada a $5.000 \mathrm{rpm}$ durante $5 \mathrm{~min}$, desprezandose o sobrenadante cuidadosamente. O material assim obtido foi dividido em duas alíquotas, uma foi analisada por exame direto ao microscópio e a outra seguiu metodologia de FAUST (centrifugação-flutuação em sulfato de zinco) [20, 21].

\section{6 - Análise estatística}

O delineamento experimental utilizado foi o inteiramente casualizado, com três tratamentos (sistemas de cultivo), 60 repetições. Os dados da avaliação física foram submetidos à análise de variância (ANOVA), para avaliar a existência de diferenças significativas. Estas diferenças foram analisadas através do teste de Tukey para comparação de médias, ao nível de erro de 5\% [8]. Na análise estatística dos resultados da avaliação microbiológica e parasitológica foi utilizado o método de qui-quadrado com nível de significância de 5\% [22].

\section{3 - RESULTADOS E DISCUSSÃo}

\section{1 - Avaliação física}

As amostras de cultivo hidropônico salientaram-se por apresentar a melhor aparência, sendo que $82 \%$ caracterizaram-se como do tipo Extra e 28\% como De Primeira, demonstrando excelente qualidade na sua forma de apresentação, sem defeitos. As amostras de cultivo orgânico classificaram-se, predominantemente, como do tipo De Primeira (67\%), 13\% como do tipo Extra e apenas
$20 \%$ como De Segunda. No cultivo tradicional, $70 \%$ das amostras avaliadas caracterizaram-se como De Segunda por apresentarem ligeira descoloração e presença de pontos escuros nas folhas externas, porém, 20\% das amostras apresentaram-se como De Primeira e 10\% como do tipo Extra.

De uma maneira geral, as alfaces caracterizaram-se por tamanhos e pesos irregulares, independentemente do sistema de cultivo (Tabela 1). As amostras de cultivo hidropônico diferiram significativamente ao nível de erro de $5 \%$, das amostras de cultivo orgânico e tradicional, apresentando menor peso total e da parte bio-comestível (folhas).

TABELA 1 - Valores médios do peso total e da parte bio-comestível de alfaces, variedade crespa, segundo diferentes sistemas de cultivo, comercializadas nos principais supermercados da cidade de Salvador (BA), no período de setembro de 2003 a junho de 2004

\begin{tabular}{cccc}
\hline Sistema de cultivo & Orgânico & Tradicional & Hidropônico \\
\hline $\begin{array}{c}\text { Peso Total (g) } \\
\text { Média }\end{array}$ & $328,08^{\mathrm{A}}$ & $300,43^{\mathrm{A}}$ & $193,56^{\mathrm{B}}$ \\
VM - Vm & $430,04-241,10$ & $552,05-138,11$ & $274,19-141,28$ \\
& & & \\
Peso parte bio-comestível (g) & $210,53^{\mathrm{A}}$ & $203,51^{\mathrm{A}}$ & $132,09^{\mathrm{B}}$ \\
Média & $304,87-145,21$ & $354,50-110,69$ & $190,15-90,93$ \\
VM - Vm & & &
\end{tabular}

VM - Valor máximo; Vm - Valor mínimo

Numa mesma linha, médias com letras em comum não diferem significativamente entre si $(\mathrm{p}<0,05)$

Quando se comparam os resultados obtidos neste trabalho com os de MARQUES et al. [15], que encontraram para alface variedade Manteiga, valores médios para peso total e parte bio-comestível, respectivamente, 229,27 g e 178,36 g, observa-se uma grande variação nestes valores, explicada em parte pelas diferenças de localidade, variedade e clima. Estes estudos comprovam que não há um padrão de qualidade quanto aos atributos físicos de peso e tamanho desta hortaliça, podendo-se encontrar uma variação muito grande nos produtos colocados à venda.

\section{2 - Avaliação microbiológica}

Em nenhuma das amostras foi detectada a presença de Salmonella sp., estando de acordo com a legislação vigente (Grupo 2.a do Anexo 1, da RDC-12 ) [4]. Todas as amostras de alface apresentaram contagem de coliformes a $35^{\circ} \mathrm{C}$ (totais) e a $45^{\circ} \mathrm{C}$ (fecais), coliformes em NMP por $100 \mathrm{~mL}$ de água. A frequiência destes contaminantes nas amostras de alfaces para cada sistema de cultivo, constam na Tabela 2.

Observou-se que houve uma relação inversa entre os percentuais de contaminação e a eficiência da $2^{\mathrm{a}}$ lavagem, ou seja, quanto maior a contaminação inicial do produto, menor a eficiência deste processo de lavagem (imersão em água com $30 \%$ vinagre comum). Assim, nas amostras de cultivo orgânico que apresentaram maior número de coliformes fecais $\left(45^{\circ} \mathrm{C}\right)$, não houve apreciável redução destes microorganismos. A freqüência de amostras que 


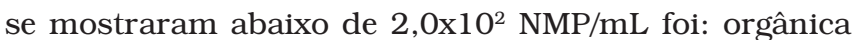
(43,33\%), tradicional $(86,67 \%)$ e hidropônica (100\%).

A contagem média global de coliformes de origem fecal apresentou diferença significativa $(\mathrm{p}>0,05)$ pelo teste de qui-quadrado ( $\aleph=22,12$ ) entre as amostras oriundas dos três sistemas de cultivo.

GUIMARÃES et al. [9] encontraram, para amostras de alface coletadas em supermercados, contagem média global de coliformes fecais igual a $3,2 \times 10^{5} \mathrm{NMP} / \mathrm{mL}$, valor pouco superior aos encontrados neste trabalho para o cultivo tradicional, na primeira lavagem.

Os resultados encontrados por MARQUES et al. [15] para amostras de alfaces comercializadas em feiras livres foram, em média, $2,4 \times 10^{6}$ e $2,4 \times 10^{3} \mathrm{NMP} / \mathrm{mL}$, respectivamente, para contagem de coliformes totais e coliformes fecais, mostrando-se bastante próximos aos encontrados neste estudo, para as amostras do cultivo tradicional.
No Brasil, embora escassos, estudos têm constatado verduras com alto grau de contaminação por coliformes fecais, sendo a água utilizada na irrigação uma importante fonte de contaminação para as hortaliças [23, 9, 16]. Esse fato poderia justificar, em parte, a alta contagem de coliformes fecais nas amostras de alfaces avaliadas neste estudo. Por outro lado, demonstra que, em algum momento, seja na produção, transporte, armazenamento ou manipulação das alfaces, houve contato dessas hortaliças com fezes de origem humana e/ou animal.

\section{3 - Avaliação microscópica}

Os resultados obtidos revelaram que, em todas as amostras, independente do sistema de cultivo, ocorreu algum tipo de contaminação. As formas parasitárias e/ou contaminantes mais freqüentes, segundo cada sistema de cultivo das alfaces, constam na Tabela 3.

TABELA 2 - Distribuição em freqüência de microbiota contaminante em amostras de alfaces, variedade crespa, segundo diferentes sistemas de cultivo, comercializadas nos principais supermercados da cidade de Salvador (BA), no período de setembro de 2003 a junho de 2004

\begin{tabular}{|c|c|c|c|c|c|c|c|c|c|c|}
\hline \multirow[b]{2}{*}{ Sistema de cultivo } & \multirow[b]{2}{*}{ Contaminantes indicadores } & & \multicolumn{7}{|c|}{ Variação na microbiota contaminante } & \multirow[b]{2}{*}{$\begin{array}{c}10^{7}-10^{8} \\
(\%)\end{array}$} \\
\hline & & & $\begin{array}{c}0-10 \\
(\%)\end{array}$ & $\begin{array}{c}10^{1}-10^{2} \\
(\%)\end{array}$ & $\begin{array}{c}10^{2}-10^{3} \\
(\%)\end{array}$ & $\begin{array}{c}10^{3}-10^{4} \\
(\%)\end{array}$ & $\begin{array}{c}10^{4}-10^{5} \\
(\%)\end{array}$ & $\begin{array}{c}10^{5}-10^{6} \\
(\%)\end{array}$ & $\begin{array}{c}10^{6}-10^{7} \\
(\%)\end{array}$ & \\
\hline \multirow{4}{*}{ Orgânico } & Coliformes Totais $\left(35^{\circ} \mathrm{C}\right)$ & 1a lavagem & - & - & - & - & 3,34 & 10 & 73,33 & 13,33 \\
\hline & & 2a lavagem & & & & 6,67 & 13,33 & 73,33 & 6,67 & \\
\hline & Coliformes Fecais $\left(45^{\circ} \mathrm{C}\right)$ & 1a lavagem & - & 13,33 & 20 & 20 & 46,67 & - & - & - \\
\hline & & 2a lavagem & 30 & 13,33 & 56,67 & - & - & - & - & - \\
\hline \multirow{3}{*}{ Tradicional } & Coliformes Totais $\left(35^{\circ} \mathrm{C}\right)$ & 1a lavagem & - & - & - & - & - & 10 & 86,67 & 3,33 \\
\hline & & 2a lavagem & - & - & - & 6,67 & 16,67 & 76,66 & - & - \\
\hline & Coliformes Fecais $\left(45^{\circ} \mathrm{C}\right)$ & 1a lavagem & - & 13,33 & 20 & 53,34 & 13,33 & - & - & - \\
\hline \multirow{5}{*}{ Hidropônico } & & 2a lavagem & 20 & 66,67 & 13,33 & - & - & - & - & - \\
\hline & Coliformes Totais $\left(35^{\circ} \mathrm{C}\right)$ & 1a lavagem & - & - & - & - & 16,67 & 60 & 23,33 & - \\
\hline & & $2^{\text {a }}$ lavagem & - & - & 10 & 26,67 & 53,33 & 10 & - & - \\
\hline & Coliformes Fecais $\left(45^{\circ} \mathrm{C}\right)$ & 1a lavagem & - & 36,67 & 63,33 & - & - & - & - & - \\
\hline & & 2a lavagem & 100 & - & - & - & - & - & - & - \\
\hline
\end{tabular}

TABELA 3 - Freqüência de formas parasitárias e outros contaminantes em amostras de alface, variedade crespa, segundo diferentes sistemas de cultivo, comercializadas nos principais supermercados da cidade de Salvador (BA), no período de setembro de 2003 a junho de 2004

\begin{tabular}{|c|c|c|c|c|c|c|c|c|c|c|c|c|}
\hline \multirow{3}{*}{$\begin{array}{l}\text { Formas parasitárias e/ou } \\
\text { contaminantes }\end{array}$} & \multicolumn{4}{|c|}{ Orgânico (n=60) } & \multicolumn{4}{|c|}{ Tradicional $(n=60)$} & \multicolumn{4}{|c|}{ Hidropônico (n=60) } \\
\hline & \multicolumn{2}{|c|}{$1^{\text {a }}$ lavagem } & \multicolumn{2}{|c|}{$2^{a}$ lavagem } & \multicolumn{2}{|c|}{$1^{\mathrm{a}}$ lavagem } & \multicolumn{2}{|c|}{$2^{\mathrm{a}}$ lavagem } & \multicolumn{2}{|c|}{$1^{\text {a }}$ lavagem } & \multicolumn{2}{|c|}{$2^{\mathrm{a}}$ lavagem } \\
\hline & $\mathrm{N}^{\circ}$ pos. & $\%$ & $\mathrm{~N}^{\circ}$ pos. & $\%$ & $\mathrm{~N}^{\circ}$ pos. & $\%$ & $\mathrm{~N}^{\circ}$ pos. & $\%$ & $\mathrm{~N}^{\circ}$ pos. & $\%$ & $\mathrm{~N}^{\circ}$ pos. & $\%$ \\
\hline Ácaros & 06 & 10 & - & - & 06 & 10 & - & - & - & - & - & - \\
\hline Insetos inteiros (Larvas) & 24 & 40 & 28 & 46,7 & 24 & 40 & 30 & 50 & 22 & 36,7 & 28 & 46,7 \\
\hline Fragmentos de insetos & 50 & 83,3 & 30 & 50 & 51 & 85 & 30 & 50 & 48 & 80 & 28 & 46,7 \\
\hline Trichostrongylus sp. & 06 & 10 & 02 & 3,3 & 04 & 6,7 & 02 & 3,3 & 02 & 3,3 & 02 & 3,3 \\
\hline Ancilostomídeos & 20 & 33,3 & 08 & 13,3 & 18 & 30 & 08 & 13,3 & 16 & 26,7 & 04 & 6,7 \\
\hline Strongyloides sp. & 08 & 13,3 & - & - & 06 & 10 & - & - & 06 & 10 & - & - \\
\hline E. nana / Endolimax sp. & 04 & 6,7 & - & - & - & - & - & - & - & - & - & - \\
\hline E. histolytical E. dispar & 06 & 10 & - & - & - & - & - & - & - & - & - & - \\
\hline E. coli / Entamoeba sp. (8N) & 02 & 3,3 & - & - & - & - & - & - & - & - & - & - \\
\hline
\end{tabular}

$\mathrm{N}^{\mathrm{o}}$ pos. = número de positivos

$(-)$ = não detectado 
Todas as amostras foram consideradas de qualidade microscópica insatisfatória, segundo a resolução 12/78 [5], que preconiza a ausência de sujidades, parasitas e larvas. A contaminação por formas parasitárias apresentou diferença significativa $(\mathrm{p}>0,05)$ pelo teste de qui-quadrado entre as amostras dos três sistemas de cultivo.

As formas contaminantes menos freqüentes foram os ácaros, presentes em 10\% das amostras do cultivo orgânico e tradicional. A presença de insetos foi observada em $40 \%$ das amostras e fragmentos de insetos no mínimo em $80 \%$ das amostras, independente do sistema de cultivo. Estes resultados diferem daqueles encontrados por GUIMARÃES et al. [9] em seu estudo sobre a freqüência de enteroparasitas em alfaces comercializadas em sacolões e feiras livres de Lavras (MG), onde relataram que, em ordem decrescente, as formas parasitárias e/ou contaminantes mais freqüentes foram: ovos de ácaros-41,7\% (50/120); ácaros-40,8\% $(49 / 120)$ e insetos-34,2\% (41/120) das amostras.

A análise das freqüências de cada tipo de helmintos (Tabela 3) mostrou predominância da ocorrência de ancilostomídeos, seguida de Strongyloides sp. e Trichostrongylus sp., nos três sistemas de cultivo de alface. Nas amostras do cultivo orgânico foram encontrados outros contaminantes, com menor freqüência, que incluíram: E. nana, E. histolytica/E. díspar e E. coli. Para todos os tipos de helmintos e protozoários intestinais encontrados, as amostras de alface de cultivo orgânico apresentaram as maiores freqüências de ocorrência, enquanto que o menor nível de contaminação foi observado nas amostras do sistema hidropônico.

Embora seja reconhecida a relevância e atualidade do problema de contaminação de hortaliças por helmintos e protozoários intestinais, são poucos os trabalhos no Brasil que relatam níveis de contaminação em hortaliças a serem consumidas cruas. GELLI et al. [7] encontraram predominância de ovos e/ou larvas de ancilostomídeos em $32,4 \%$ das amostras de alfaces comercializadas no município de São Paulo.

OLIVEIRA \& GERMANO [20] também encontraram, em seu estudo com alfaces crespas e lisas comercializadas na região metropolitana de São Paulo (SP), maior ocorrência de ancilostomídeos nas amostras. Os autores relataram a recuperação de ovos de trichostrongilídeos, comprovando a ocorrência de contaminação das hortaliças por fezes de animais ruminantes, que comumente são utilizadas na adubação de hortas. Este fato poderia justificar, em nosso estudo, a maior freqüência de trichostrongilídeos nas amostras de alfaces do cultivo orgânico e tradicional, que freqüentemente utilizam dejetos orgânicos para adubação.

Todos os enteroparasitas recuperados apresentam importância para a saúde pública. A maioria indica contaminação fecal de origem humana e/ou animal, uma vez que apresenta espécies de ocorrência no homem, nos animais ou em ambos [11].

Observa-se que há similaridade entre os resultados deste estudo e os observados por outros autores no país
[7, 12, 16, 19, 20], embora possam apresentar variação na espécie ou freqüência de enteroparasitas, explicada, em parte, pela localidade, tipos de hortaliças e metodologia utilizada no exame parasitológico.

As diferenças entre os níveis de contaminação dos três sistemas de cultivo de alfaces, parecem estar associadas, fundamentalmente, com as condições sanitárias do ambiente em que são cultivadas, diferentes em cada sistema produtor, de acordo com as práticas de cultivo utilizadas $[7,16]$. Deste modo, os percentuais de contaminação observados no sistema de cultivo hidropônico de alfaces, além de serem injustificados, podem ser atribuídos, entre outros fatores, às condições higiênico-sanitárias da água, pois neste sistema as hortaliças recebem os nutrientes previamente dissolvidos em água, não sendo plantadas em terra, que é reconhecidamente portadora de inúmeros parasitas e contaminantes.

Dada a constatação da permanência de enteroparasitas patogênicos nas amostras de água da segunda lavagem (água $+30 \%$ vinagre) das alfaces dos três sistemas de cultivo e dada a importância que estes resultados revelaram sob o ponto de vista de saúde pública, pois trata-se de procedimento comum de lavagem doméstica, torna-se evidente a necessidade da adoção de medidas de fiscalização em todo o processo produtivo e de comercialização dessa hortaliça.

\section{4 - CONCLUSÕES}

As alfaces de cultivo hidropônico foram inseridas como hortaliças da classe Extra e as de cultivo orgânico e tradicional na Primeira e Segunda classe, respectivamente. Apresentaram o menor peso total e da parte bio-comestível, diferindo significativamente das alfaces de cultivo orgânico e tradicional que apresentaram valores maiores.

Nas condições em que foi desenvolvido este estudo, conclui-se que, as amostras de alfaces de cultivo orgânico, tradicional e hidropônico, comercializadas nos principais supermercados de Salvador (BA), apresentaram baixo padrão higiênico, evidenciado pela presença de formas parasitárias de origem humana e/ou animal e alta contaminação por coliformes fecais.

A constatação da ocorrência de enteroparasitas em alfaces e a permanência de alguns destes patógenos na água de lavagem, segundo procedimentos domésticos comuns e, dada à importância que apresenta para saúde pública, há necessidade de adoção de medidas, por parte dos órgãos de vigilância sanitária, para melhoria da qualidade higiênica dessa hortaliça tão consumida pela população.

\section{5 - REFERÊNCIAS BIBLIOGRÁFICAS}

[1] ABIA - ASSOCIAÇÃO BRASILEIRA DAS INDÚSTRIAS DA ALIMENTAÇÃO. Compêndio da Legislação de Alimentos. Consolidação das normas e padrões de alimentos. Órgão Técnico e Consultivo do Poder Público. Atos do Ministério da Saúde. Decreto $n^{\circ} 54.541$, de 22 de outubro de 1964. Última revisão em 1996. v. 1 e 1/A. 
[2] Anônimo. Orgânico ou hidropônico? Não faça confusão! Disponível em http:// www.hortaearte.com. br/orghidro.htm. Acesso em $1^{\circ}$ fev. 2004.

[3] A.O.A.C. - ASSOCIATION OF OFFICIAL ANALYTICAL CHEMISTS. Official methods of analysis of the AOAC International. $17^{\text {th }}$ ed., Washington: A.O.A.C., 2000, 1.115 p.

[4] BRASIL. Ministério da Saúde. Secretária Nacional de Vigilância Sanitária. Resolução no 12 , de 2 de janeiro de 2001. Dispõe sobre padrões microbiológicos. Diário Oficial [da] República Federativa do Brasil. Brasília (DF), 10 jan. 2001. Seção I, p. 48.

[5] BRASIL. Resolução Normativa no 12/78. Aprova Normas Técnicas Especiais do Estado de São Paulo, relativa a alimentos e bebidas. Diário Oficial [da] República Federativa do Brasil, Brasília, 24 julho de 1978, Seção I, pt. I, p. 11.525.

[6] FERNANDES, A.A.; MARTINEZ, H.E.P.; PEREIRA, P.R.G.; FONSECA, M.C.M. Produtividade, acúmulo de nitrato e estado nutricional de cultivares de alface, em hidropônia, em função de fontes de nutrientes. Horticultura Brasileira, Brasília, v. 20, n. 2, p. 195-200, 2002.

[7] GELLI, D.S.; TACHIBANA, T.; OLIVEIRA, J.R.; ZAMBONI, C.Q.; PACHECO, J.A.; SPITERI, N. Condições higiênico-sanitárias de hortaliças comercializadas na cidade de São Paulo (SP), Brasil. Revista do Instituto Adolfo Lutz, São Paulo, v. 39, p. 37-43, 1979.

[8] GOMES, F.P. Curso de estatística experimental. $13^{\mathrm{a}}$ ed., São Paulo: Nobel, 1990, 468 p.

[9] GUIMARÃES, A.M.; ALVES, E.G.L.; FIGUEIREDO, H.C.P; COSTA, G.M.; RODRIGUES, L.S. Freqüência de enteroparasitas em amostras de alface (Lactuca sativa) comercializadas em Lavras, Minas Gerais. Rev. Soc. Bras. Med. Trop., v. 36, n. 5, p. 132135, 2003.

[10] HAMERSCHMIDT, I. Agricultura orgânica: conceituações e princípios. In: Anais do $38^{\circ}$ Congresso Brasileiro de Olericultura. Petrolina (PE): ART \& MÍDIA, 1998. cd-rom.

[11] HEALY, G.R.; JACKSON, G.J.; LICHTENFELS, J.R.; HOFFMAN, G.L.; CHENG, T.C. Foodborne parasites. In: SPECK, M.L., ed. Compendium of methods for the microbiological examination of foods. $2^{\text {nd }} \mathrm{ed}$. Washington D.C., American Public Health Association, 1984, p. 542-556.

[12] HERNANDES, N.; CIMERMAN, B.; FERNANDES, M.F.P.; FERRAZ, C.A.M.; ARAÚJO, A.L.; SILVA, C.A. Estudo da contaminação de verdura no Município de Biritiba Mirim. In: Congresso Brasileiro de Parasitologia, 6, Belo Horizonte, 1981. Resumos. Belo Horizonte, Sociedade Brasileira de Parasitologia, 1981, p. 219.

[13] KRAMER, A.; TWIGG, B.A. Quality control for the food industry. $3^{\text {rd }}$ ed. Westport: AVI, 1973, $556 \mathrm{p}$.
[14] LEITÃO, M.F.F. Controle higiênico-sanitário de alimentos. Boletim ITAL, Campinas, v. 18, n. 2, p. 201-226, 1981.

[15] MARQUES, M.A.; SILVA, S.M.; MARTINS, L.P.; SANTOS, J.G. Qualidade física e microbiológica de hortaliças comercializadas na feira livre do município de bananeiras (PB). In: Congresso Brasileiro de Ciência e Tecnologia de Alimentos, 18, Porto Alegre, 2002. Resumos. Porto Alegre, Sociedade Brasileira de Ciência e Tecnologia de Alimentos, 2002, p. 125.

[16] MARZOCHI, M.C.A. Estudo dos fatores envolvidos na disseminação dos enteroparasitas. II - Estudo da contaminação de verduras e solo de hortas na cidade de Ribeirão Preto, São Paulo, Brasil. Rev. Inst. Med. Trop., São Paulo, v. 19, p. 148-155, 1977.

[17] MESQUITA, V.C.L.; SERRA, M.B.; BASTOS, O.M.P.; UCHÔA, C.M.A. Contaminação por enteroparasitas em hortaliças comercializadas nas cidades de Niterói e Rio de Janeiro, Brasil. Revista da Sociedade Brasileira de Medicina Tropical, v. 32, p. 363-366, 1999.

[18] MIYAZAWA, M.; KHATOUNIAN, C.A.; ODENATH-PENHA, L.A.; Teor de nitrato nas folhas de alface produzida em cultivo convencional, orgânico e hidropônico. Agroecologia Hoje, n. 2, p. 23, 2001.

[19] MOTA, C.S.S.; ELIAS, A.; MIKOSZEWSKA, I.; VIEIRA, H.R.A.; PICKET NETO, J.; VASQUES, R.M.R.; ALMEIDA, A.A.; GAISSLER, M.S.; BEATRIZ, R.; MOTA, R.M.T.C.S. Condições higiênico-sanitárias de hortaliças comercializadas em Curitiba (PR), Brasil. In: Congresso Brasileiro de Ciência e Tecnologia de Alimentos, 6, Brasília, 1983. Resumos. Brasília, Sociedade Brasileira de Ciência e Tecnologia de Alimentos, 1983, p. 125.

[20] OLIVEIRA, C.A.F.; GERMANO, P.M.L. Estudo da ocorrência de enteroparasitas em hortaliças comercializadas na região metropolitana de São Paulo (SP), Brasil. I - Pesquisa de helmintos. Rev. Saúde Pública, v. 26, n. 4, p. 283-289, 1992.

[21] REY, L. Parasitologia. $2^{\mathrm{a}}$ ed. Rio de Janeiro: Guanabara Koogan, 1991, 117 p.

[22] SAMPAIO, I.B. Estatística aplicada à experimentação animal. Fundação de Ensino e Pesquisa em Medicina Veterinária e Zootecnia. Belo Horizonte, 1988, 51 p.

[23] TAKAYANAGUI, O.M.; OLIVEIRA, C.D.; BERGAMINI, A.M.N.; CAPUANO, D.M.; OKINO, M.H.T.; FEBRÔNIO, L.H.P.; SILVA, A.A.M.C.C.; OLIVEIRA, M.A.; RIBEIRO, E.G.A., TAKAYANAGUI, A.M.M. Fiscalização de verduras comercializadas no município de Ribeirão Preto, São Paulo. Revista da Sociedade Brasileira de Medicina Tropical, v. 34, p. 37-41, 2001.

[24] VANDERZANT, C.; SPLITTSTOESSER, D.F. Compendium of methods for the microbiological examination of foods. Washington: American Public Health Association, 1992. 